\title{
APPLYING SOLVABILITY THEOREMS FOR MATRIX EQUATIONS
}

\section{Z. MOUSAVi AND F. MirZAPOUR}

Abstract. In this paper, using solvability theorems for matrix equations, generally applicable results are proved for the existence of positive semidefinite or asymptotically positive semidefinite solution. In the following, a question about the matrix equation $f(A) X+X f(A)=A B+B A$ is answered. This question was asked, first by Chan and Kwong [6] and then by Furuta [7].

Mathematics subject classification (2020): 15A24.

Keywords and phrases: Matrix equation, solvability theorem, positive definite solution, Farkas lemma.

\section{REFERENCES}

[1] A. Ben-IsRael, Linear Equations and Inequalities on Finite Dimensional, Real or Complex, Vector Spaces: A Unified Theory, J. Math. Anal. Appl. 27, 367-389 (1969).

[2] E. V. Belmega, S. LAS Aulce And M. DeBBAh, A Trace inequality for positive definite matrices, Journal of inequalities in pure and applied mathematics, Vol. 10 (2009), Issue 1, Article 5, 4 pp.

[3] A. Berman AND A. Ben-IsRael, More on linear inequalities with applications to matrix theory, J. Math. Anal. Appl. 33 (1971), 482-496.

[4] A. BERMAN AND A. BEN-ISRAEL, Linear Equations over Cones with Interior: A Solvability Theorem with Applications to Matrix Theory, Linear Algebra Appl. 7 (1973) 139-149.

[5] R. Bhatia, Matrix Analysis, Grad. Texts in Math. 169, Springer-Verlag, New York, 1997.

[6] N. N. Chan AND M. K. Kwong, Hermitian matrix inequalities and a conjecture, Amer. Math. Monthly 92 (1985), 533-541.

[7] T. Furuta, Positive semidefinite solutions of the operator equation $\sum_{j=1}^{n} A_{n-j} X A_{j-1}=B$, Linear Algebra Appl. 432 (2010) 949-955.

[8] P. Lancaster and L. Rodman, Algebraic Riccati Equations, Oxford University Press, New York, 1995.

[9] T. MorI, Comments on "A Matrix Inequality Associated with Bounds on Solutions of Algebraic Riccati and Lyapunov Equation”, IEEE Trans. Automat. Contr., vol. AC-33, p. 1088, Nov. 1988.

[10] A. M. RAN AND M. C. B. ReuRINGS, The symmetric linear matrix equation, The Electronic Journal of Linear Algebra 9 (2002) 93-107. 